\title{
Balkanologie
}

Balkanologie Revue d'études pluridisciplinaires

Vol. IV, $\mathbf{n}^{\circ} 2$ | 2000

Volume IV Numéro 2

\section{La maisnie diffuse, du communisme au capitalisme : Questions et hypothèses}

\section{Vintilă Mihăilescu}

\section{(2) OpenEdition}

12 Journals

Édition électronique

URL : http://journals.openedition.org/balkanologie/334

DOI : 10.4000/balkanologie.334

ISSN : 1965-0582

Éditeur

Association française d'études sur les Balkans (Afebalk)

Édition imprimée

Date de publication : 1 décembre 2000

ISSN : 1279-7952

\section{Référence électronique}

Vintilă Mihăilescu, "La maisnie diffuse, du communisme au capitalisme : Questions et hypothèses », Balkanologie [En ligne], Vol. IV, n² 2 | 2000, mis en ligne le 30 mai 2008, consulté le 17 décembre 2020. URL : http://journals.openedition.org/balkanologie/334; DOI : https://doi.org/10.4000/balkanologie. 334

Ce document a été généré automatiquement le 17 décembre 2020.

(c) Tous droits réservés 


\title{
La maisnie diffuse, du communisme au capitalisme : Questions et hypothèses
}

\author{
Vintilă Mihăilescu
}

1 Alors que je travaillais pour un projet PHARE de développement local, je me suis trouvé dans la position de mettre sur pied, avec les gens d'un village, un projet de construction d'une petite fabrique de produits laitiers et d'en obtenir le financement. Pour gérer le projet et ensuite le fonctionnement de la fabrique, les gens du village devaient s'organiser en association. Ce qui n'arriva pas et le financement fut retiré. Révolté, de passage dans le village, j'en ai demandé la raison. «L'explication » qu'on a fini par me donner m'est restée en mémoire: «Écoute, Monsieur, quitte à se faire rouler entre nous, on finit quand même par se débrouiller !» (c'est-à-dire sans toi et tes projets). Ce n'était pas un village vieux et isolé et ses habitants étaient loin de vivre dans la misère. Et l'expérience me disait que ce n'était pas un cas isolé non plus. Alors?

Depuis, j'ai essayé de construire ma propre explication, pour comprendre leur explication et tout le petit monde qu'elle exprime d'une manière exemplaire. Ce qui suit sont des réflexions plus ou moins poussées sur ce sujet.

\section{Un cas peu singulier}

3 Cette simple phrase pose au moins trois questions fondamentales, dont la réponse n'est pas à portée de main :

4 1) qui est ce nous? Autrement dit, quel est le système de relations dans et par lequel on peut « se débrouiller »?

5 2) comment arrivent-ils à se débrouiller ? Quel est donc le système de production mis en place pour y parvenir? 
6 3) quelle est la raison de ce choix, qui les a poussés à préférer " se tromper entre eux » au lieu d'avoir à gérer ensemble une petite entreprise qui leur était extrêmement utile?

7 Pour essayer de répondre, il faut replacer ce cas particulier dans son contexte plus large, qui est le monde rural roumain et sa longue durée. Mais avant d'y venir, quelques informations sur le contexte restreint du village même.

8 Pucheni est un village de 2365 habitants, dans une région de collines, près de la grande route qui lie Tîrgoviste et Câmpulung, deux villes industrialisées surtout pendant le communisme, où une bonne partie des habitants travaille comme ouvriers. Les 600 hectares de pâturage, les 16000 pruniers et les 7000 pommiers représentent des ressources supplémentaires pour la population.

Parmi les données statistiques d'un questionnaire sociologique appliqué au niveau du village, quelques faits sont à retenir. Ainsi, par exemple, $41 \%$ des maisnies ont pratiqué pendant l'année 1998, d'une manière ou d'une autre, l'échange en nature. Par contre, les investissements agricoles sont très faibles et ne dépendent, statistiquement, ni de l'âge, ni du niveau d'instruction, ni de la surface de terre en propriété, mais... du nombre de chambres des maisnies. Ces dernières sont souvent assez grandes, avec un nombre démesuré de chambres par rapport aux nombre d'habitants et, ce qui est extrêmement significatif, leur dotation en téléphone, téléviseur, voiture, machine à laver, etc., ne dépend pas statistiquement des revenus déclarés de la famille. Ce n'est donc pas en fonction de ce qu'ils gagnent que les gens vont avoir une maison plus ou moins « riche».

Une explication anecdotique de ce mystère nous a été offerte par un officiel du village, qui venait de bâtir une maison en ville pour son garçon (d'une dizaine d'années) à coup d'eau-de-vie : tout avait été " payé » en nature et quand il n'a plus eu d'eau-de-vie, il en a emprunté à son beau-père, en lui promettant en échange de l'aider aux travaux agricoles en été.

11 Toute cette alchimie qui transforme eau-de-vie en maison porte, habituellement, un nom : économie informelle. Mais ce simple nom ne suffit pas pour comprendre tout ce qui se cache derrière.

\section{"Quitte à se tromper entre nous..." Un système de relations}

C'est qui, nous? - avons nous demandé. Qui est ce « sujet collectif » diffus et pourtant efficace?

Pour essayer de comprendre, il faut placer la question dans la longue durée et remonter, probablement, jusqu'à la obşte devălmăşie, cette structure économique et politique typique des anciens villages roumains, apparentée mais différente de ses voisines, le mir russe et la zadruga des Slaves du sud. Sans entrer dans les détails, il suffit pour notre propos de préciser que cette communauté indivise de la obşte est restée un vrai sujet juridique jusqu'au début du XXème siècle. Ainsi, par exemple, " le décret de Décembre 1918 ne prévoyait aucune disposition de mise en propriété (...). La terre expropriée [et donnée aux paysans] devait être travaillée par l'ensemble de la obște ${ }^{1}$, qui en était ainsi le vrai sujet juridique. C'est ainsi qu'au long d'un siècle, les villageois 
n'ont fait qu'interpréter dans un esprit devălmăşie toutes les reformes de l'État de telle manière que, paradoxalement, la série des mises en propriété qui ont suivi n'ont pas engendré une montée de l'esprit de propriété individuelle mais ont conduit, tout au contraire, vers une reprise de la croyance dans une indivision communautaire de toutes les terres du pays auxquelles eux, les villageois, auraient le droit d'usage selon leur besoin, en payant bien sûr les impôts et les redevances².

Cette organisation indivise quitte, avec le temps, le niveau de la communauté toute entière, s'organise en lignages (spiçe de neam) et fini par occuper surtout l'espace de la maisnie. On peut dire ainsi que c'est la maisnie et non pas l'individu qui constitue la dernière étape de dissolution de la obște devălmăşie .

D'une manière typique, la maisnie est composée par les parents, les enfants non-mariés et, le cas échéant, la famille du dernier né (qui reste dans la maison parentale) et qui sont les propriétaires et producteurs devălmăşie(en indivision) des biens de la maisnie, celle-ci étant ainsi « l'unité de production et non pas seulement de consommation ${ }^{4}$ de la vie villageoise.

Dans le cas de la maisnie, « la notion juridique de propriété est remplacée par un fait : la prestation du travail en commun $»^{5}$. Cette propriété commune de facto (et non pas individuelle de jure) va être répartie d'une manière égalitaire entre tous les descendants à leur mariage, avec interdiction coutumière d'aliénation. «Elle ne veut pas donner toute la terre à son fils, qui habite avec elle et travaille la terre, car elle a encore deux enfants à Bucarest et elle doit faire leur part à eux aussi, c'est la loi, elle ne peut pas les laisser sans rien »- nous expliquait encore, il y a quelques années, une paysanne de la plaine danubienne.

17 Tout en restant le modèle, cette structure est devenue de plus en plus rare, surtout avec le communisme, à cause des émigrations industrielles et du vieillissement démographique des villages. Dès lors, la maisnie commence à être traversée par des tendances opposées, centripètes et centrifuges, se recomposant sans cesse en fonction des enjeux de mobilité et de stabilité de ses membres. Divisée, grosso modo, entre une partie fixe, qui reste au village, et une partie mobile, travaillant ou même habitant dans les villes, une bonne partie des maisnies se recompose en tant que «maisnies mixtes diffuses $»^{6}$. «Délocalisée », pour ainsi dire, ne pouvant plus garder, la plupart du temps, son unité structurale, la maisnie s'efforce de limiter les distances pour sauvegarder au moins la proximité physique et sociale, en faisant pression sur les choix professionnels et matrimoniaux de ses membres. Même séparées, des unités familiales essayent de partager l'espace et même le budget, quand elles sont dans le même village ou, émigrées en ville, de se refaire, à coup de changements de domicile, une proximité d'habitation familiale. On peut parler ainsi, dans beaucoup de cas, d'une "résidence diffuse ", plus ou moins partagée par une unité familiale, dont les membres passent des heures ou des jours ici ou là, selon les besoins des uns et des autres. "On venait d'avoir la deuxième fille et on avait droit à trois chambres, mais dans le 5ème arrondissement. Moi je voulais vivre ici, dans le 6ème, pour être plus près de mes parents » - expliquait un intellectuel de Bucarest, d'origine villageoise. Ou bien un autre locataire de bloc qui racontait : «Ma belle-mère vient souvent chez nous, il ne passe pas une semaine sans qu'elle nous rende visite. Quelquefois, elle reste la nuit, ou même plusieurs nuits chez nous $»^{7}$. D'une manière assez générale, les enfants des villes passent le plus clair de leurs vacances chez les grands-parents des villages et ceux-ci viennent passer (moins souvent!) la saison froide en ville, l'ensemble (en principe) de la famille se réunit pour 
les travaux agricoles et pour les fêtes, on donne, on prend et on partage. Les frontières entre moi et nous, le mien et le nôtre sont ainsi assez diffuses elles aussi, avec, à l'extrême de la devălmăşie, un air de promiscuité. Quelquefois, une jeune famille essaie de rompre ces liens (les «familles mobiles» dont parle Kideckel ${ }^{8}$ ) et sort, plus ou moins, de cette gravitation. À l'autre extrême, le réseau s'élargit, assimilant de nouveaux voisins ou collègues de service par l'institution de la parenté symbolique ou simplement en en faisant des invités systématiques aux réunions de famille.

18 Après la chute du communisme, ces réseaux vont être réinvestis et adaptés aux nouvelles conditions. Composant maintenant aussi avec les nouvelles ressources d'une économie "libérée ", pour ne pas dire de marché, les réseaux centrés sur la maisnie se développent, se différencient, sans pour autant abandonner une certaine logique sociale qui leur est propre.

19 Il en résulte une telle diversité qu'on aurait tort de vouloir la systématiser selon des critères morphologiques. La maisnie diffuse n'est pas une structure locative, mais un réseau, ou, mieux encore, un ensemble de stratégies dont le point commun est la construction sur un choix de parenté (réelle et même "assimilée »), plus ou moins dispersée dans le territoire, des réseaux capables d'assurer, en partie au moins, la fonction d'unité de production et de consommation de la maisnie.

20 Cette unité, aussi « diffuse » soit-elle, rapproche les catégories sociales et devient ainsi trans-catégorielle. Paysans et ouvriers, villageois et citadins, etc., échangent et partagent d'une manière systématique à travers ces réseaux de la maisnie diffuse : ils forment des nous, qui ne sont plus à $100 \%$ ni paysans, ni ouvriers, ni villageois, ni citadins (dans un village où nous avons fait du terrain pendant l'été, la population doublait chaque fin de semaine avec les parents venus des villes plus ou moins voisines, de sorte que nous avons été amenés à nous poser la question de la " vraie " structure démographique du village). Il devient ainsi douteux, d'un point de vue méthodologique, de continuer à mener des analyses sociales suivant strictement les oppositions villevillage ou paysan-ouvrier ou de choisir entre «la mort du paysan » et «le retour du paysan». Mais surtout, il devient peu éclairant de vouloir trancher entre individualisme et collectivisme. "Il n'y pas de pire et stérile distinction dans la sociologie primitive que celle entre individualisme et communisme » - nous avertissait déjà Malinowski dans Coral Gardens and Their Magic. Et Chris Hann va plus loin :

21 Le refus de l'alternative communisme / individualisme par Malinowski devrait être élargi au-delà des sociétés qu'il avait en tête. C'est important de regarder derrière les rhétoriques qui se confrontent dans notre temps pour analyser par contre les réalités $\mathrm{du}$ changement des exigences collectives et personnelles avant, pendant et après le socialisme. (...) Dans le contexte de la décollectivisation en Europe de l'Est, son invitation de prendre en compte "la relation entre les exigences collectives et personnelles" reste un point de départ valable, tout comme son conseil d'explorer les usages de la terre dans le sens large, "au-delà du point de vue légal"9.

Propos essentiel si on veut comprendre la maisnie diffuse, ce système de relations où les gens forment des réseaux de solidarité "quitte à se tromper" entre eux et à poursuivre chacun son intérêt.

23 Une question essentielle s'impose ainsi au coeur de la maisnie diffuse : celui de la confiance. 

devălmăşie, on passe de plus en plus (et cela déjà sous le communisme) à celle de omenie (oameni de omenie ou oameni de treabă: plus ou moins «homme-comme-il-faut»), qui définit une condition relationnelle des personnes auxquelles on peut faire confiance dans les échanges réciproques.

Les enjeux économiques ont amené les gens à se positionner réciproquement selon une échelle de confiance et de coopération. À un extrême se trouvaient les oameni 
de treabă, auxquels on peut faire confiance, laborieux et coopérants. Au milieu de l'échelle, se trouvaient les şmecheri (malins), auxquels on ne pouvait pas faire confiance mais qui restaient passablement menaçants. À l'autre extrême, on trouvait les afacerişti ou bişniŢari (spéculateurs), qui se servaient des autres pour leur propre intérêt et pensaient tout le temps à faire du profit aux dépens des autres. (...) Être classifié ainsi impliquait la perte de confiance des autres et, du même coup, de leur coopération ${ }^{11}$.

Enfin, les règles de la réciprocité communautaire changent elles aussi. Le don de noce en est un exemple typique. Selon la règle générale, celui-ci engage la maisnie et non pas l'individu et est destiné à garantir, par la communauté, une égalité des chances des couples à leur mariage (et non pas des individus à leur naissance). À Pucheni, par exemple, tout le monde gardait encore des "cahiers de noces", où la famille notait toutes les participations aux mariages du village et la valeurs des dons faits aux mariés. Pour s'assurer du maintien de la réciprocité dans les conditions récentes d'inflation, les calculs ont été convertis en dollars. Cette "comptabilisation» de la réciprocité permettait aussi de juger sur l'intérêt qu'on a de rester ou non dans ce circuit de réciprocité et il y avait des familles qui, tout compte fait, préféraient se retirer.

Pour donner un nom à toute cette dynamique sociale, on pourrait parler d'un passage de la réciprocité normative à une réciprocité sélective, matière plutôt à stratégie individuelle qu'à gestion communautaire. Mais pourquoi ce choix ? De toute évidence parce qu'il est disponible et utile: il permet de "se débrouiller ", d'assurer sa place dans la société. Rester dans un réseau de solidarité, quitte à le reconstruire de temps en temps, est considéré, globalement et à long terme, plus avantageux et plus sûr que de s'attaquer à la vie en solitaire. On fait ainsi confiance au réseau de réciprocité, tout en se méfiant des gens qui le composent. On ne renonce pas aux avantages des relations fondées sur le don et le contre-don, tout en essayant d'avoir un maximum de contrôle et d'initiative par rapport à ces relations et se sentir ainsi mettre du jeu. Il n'est alors pas surprenant de voir les réticences de ces gens par rapport à des règles de jeu encore mal connues ou qui les mettent hors-jeu. Il ne s'agit donc pas tant de conservatisme ou d'inertie sociale que de créativité dans des contextes donnés.

Par ailleurs, cette réciprocité sélective articule sui generis individualisme et collectivisme. Si on garde «l'atomisation» comme métaphore d'une société individualiste, alors on devrait parler dans ce cas-là de "molécularisation ». L'individu n'est pas «libéré » de son groupe, mais essaye de contrôler le choix de celui-ci. Au niveau global de la société, il en résulte un tissu social fragmenté, fait de nous relativement dépendants à l'intérieur mais relativement indépendants les uns des autres et réticents à se faire intégrer dans des projets de société plus vastes. Ce qui pose, comme le remarquait Kideckel ${ }^{12}$, un grave problème politique, touchant l'état même de la nation.

\section{“...on finit quand même par se débrouiller". Un « système de production »}

Les réseaux de réciprocité sélective aide à se débrouiller, avons-nous dit. Mais comment parvient-on concrètement à se débrouiller?

La particularité économique de ce système consiste dans les échanges et leurs manières de composer avec les ressources particulières auxquelles les participants ont accès du 
fait de leur occupation spécifique. Car tous les participants ont des occupations précises qui, malgré tout, ne leur sont pas suffisantes pour « se débrouiller ».

La forme d'échange la plus simple et la plus répandue est celle qui découle de l'ancienne devălmăşie familiale, reconstruite à l'échelle de la maisnie diffuse. Il s'agit, pour l'essentiel, d'échanges de redistribution des ressources à l'intérieur du réseau. À Crasani, par exemple, un village assez pauvre de la plaine danubienne, la plupart des familles a des enfants ou d'autres parents dans les villes avoisinantes: « Tout le monde ici, dans cette rue, tous ont des enfants à Bucarest. Ils ont des enfants dans les villes et ils viennent et leur apportent... ». Les échanges vont dans les deux sens : «Bon, il y a mon beau-frère, le cousin de Bucarest, il vient lui aussi, qu'est-ce qu'ils peuvent faire? Son frère a trois enfants, mon frère de Giurgiu a sa femme au chômage, je dois l'aider lui aussi; celui de Slobozia, n'est-ce pas, il vient chez moi, je lui donne une poule, du lait, ce que je trouve dans la maisnie $\aleph^{13}$. L'unité de production et de consommation de la maisnie se refait ainsi d'une certaine manière, au-delà des distances qui séparent ses membres. Un ouvrier de Bucarest, par exemple, résumait ainsi ses relations avec la campagne: «Nous, quand on va à la campagne, on travaille. On n'achète jamais de la farine, des pommes de terre, des tomates... Mon frère a toujours aimé la terre et les chevaux. Alors moi, je fais les réparations, l'installation électrique, sanitaire et ainsi de suite. Et lorsqu'on rentre à Bucarest, on apporte en gros, comme on dit, tout ce qu'il nous faut... $»^{14}$. Dans tous ces cas, il s'agit d'une redistribution des ressources par une reproduction des relations à l'intérieur d'un réseau de parenté. Et cette sorte de devălmăşie familiale est d'autant plus forte que le niveau de pauvreté est plus poussé. Stahl le constatait déjà dans les années 30 : «Plus on descend dans le niveau de pauvreté, plus la devălmăşie familiale apparait d'une manière plus claire et plus les villageois sont capables de la présenter théoriquement d'une manière plus précise $»^{15}$.

Quand la maisnie dispose de ressources plus grandes ou plus "rares", de restreinte, cette redistribution devient élargie et s'exerce aussi à l'extérieur de la maisnie. Ainsi, le camion de bois dont dispose le garde forestier peut se transformer en camion de pommes de terres offert par le paysan du village d'à côté, ou la production d'eau-de-vie du terroir en maison dans la ville, etc., tout cela à travers des réseaux d'échange plus ou moins complexes. Souvent, on a du mal à distinguer cette redistribution élargie des échanges commerciaux, si ce n'est le fait qu'elle évite par définition les voies du marché (y compris les taxes afférentes !).

Un type différent d'échange est celui qu'on peut qualifier de coopératif. On se met ensemble pour bénéficier des outillages agricoles de l'un et de la voiture de l'autre, pour aller à tour de rôle vendre les pommes au marché, etc., comme c'était le cas à Voineşti par exemple, un riche village célèbre pour ses pommes ${ }^{16}$. Par ailleurs, ces échanges coopératifs ne se transforment jamais en vraies associations formelles et ne constituent pas l'objet de calculs précis.

Mais il ne faut pas se méprendre: il n'y a pas (sauf exception) une "pureté » des réseaux et des types d'échange! La tendance est plutôt au "métissage " et l'idéal semble être de combiner, à petite échelle, échanges et ventes, "économie informelle " et économie de marché. À Pucheni, par exemple, la dotation de la maison et la satisfaction par rapport à la vie (qu'on peut considérer des indicateurs de réussite) son liés statistiquement à la pratique de la vente au marché et à celle des échanges en nature. Autrement dit, pour réussir, il faut combiner les deux. 
39 L'ensemble de ces échanges limités dans le temps et l'espace n'est pas sans conséquences au niveau de la société toute entière. Il en résulte une dynamique plus vaste, qu'on peut présenter, grosso modo, comme échanges public-privé et sans laquelle on aurait du mal à comprendre « le pays réel » du temps du communisme et même après.

Un bon exemple dans ce sens-là est ce qu'on peut appeler le phénomène social total du vol et contre-vol.

41 D'une part, nationalisation et collectivisation ont fait de la propriété privée une propriété collective, « du peuple ». Plus tard, avec les « contrats », la production de la maisnie devait être vendue dans sa plus grande partie à l'État, à des prix modiques, pour « le bien du peuple » dans son ensemble. D'une manière plus "subtile », chaque instance du pouvoir avait ses villages et / ou maisnies de "protocole", autant de sources de produits et services «bénévoles» pour entretenir les privilèges d'une nomenclature locale et / ou centrale et ses besoins de fonctionnement. En revanche, on fermait les yeux par-ci, par-là, permettant aux gens de refaire leur petite richesse, on facilitait des services institutionnels auxquels on avait difficilement accès, etc. Ce type d'échange se poursuivait aussi à petite échelle, entre individus, les produits de la maisnie entrant directement ou par intermédiaires dans les poches des chefs d'entreprise, qui, en revanche, patronnaient le vol des produits qui rentraient dans la maisnie. Le vol des institutions nationales était devenu une institution nationale du vol. Un paysan de Crăsani en résumait la philosophie dans ces termes : "Le loup, d'où il court, de là il doit se nourrir. Moi, donc, j'étais au secteur [zootechnique] et je ne prenais pas avec le sac, je prenais comme ça, avec les poings. Ceux qui travaillaient aux champs, ils en prenaient avec le sac. Mais moi, là-bas, à mon poste, je donnais à manger à la vache et je mangeais moi aussi. C'est équitable, n'est-ce pas?».

Ce vol et contre-vol touchaient aussi des domaines plus « ineffables ", comme la gestion du temps, par exemple. Ainsi, à la « nationalisation du temps » pratiquée par le régime communiste, dont parle Katherine Verdery, correspondait une «privatisation du temps " pratiquée par les citoyens ${ }^{17}$. Dans ce sens, à part les fameuses "pauses café ", j'ai pu observer dans la ville industrielle de Rovinari, par exemple, que les taux de productivité et d'absentéisme du grand combinat minier fluctuaient en fonction du calendrier des travaux agricoles : le «temps industriel» et " public » était volé, pour ainsi dire, par l'agriculture privée.

43 À travers les individus et leurs réseaux trans-catégoriels d'appartenance, un échange « informel » et généralisé se met ainsi en place entre maisnies et institutions et, d'une manière plus globale, entre agriculture et villages d'une part, et industrie et villes d'autre part. C'est ce que Gerald Creed appelle " the domestication of industry » pendant le communisme ${ }^{18}$, c'est-à-dire un relatif renversement des priorités politiques de l'industrialisation et de l'urbanisation par les pratiques sociales de ces maisnies diffuses d'origine rurale-agraire (« en bonne partie dans l'espace social défini par la maisnie », précise Creed).

Quoique le rôle de l'individu fût diminué, les réseaux sociaux de la maisnie étaient aussi importants comme toujours. En fait, ces réseaux médiaient la lutte entre les maisnies et l'État. Les individus représentant chacune de ces deux institutions utilisaient les réseaux pour s'adapter aux demandes et restrictions de l'autre: les membres de la maisnie pour garantir l'accès aux ressources de l'économie socialiste et les agents de l'État pour garantir une production adéquate dans les institutions socialistes ${ }^{19}$. 
des observations semblables à travers l'ensemble du pays. À Pucheni, par exemple, nous avons assisté à la scène suivante : un paysan qui travaillait son carré de pommes de terres, se fait interpeller par un autre villageois qui revenait de la ville où il travaillait : «Pourquoi tu te crèves avec ton lopin? Avec l'argent que tu y mets, je t'achète de la ville toutes tes pommes de terre et toi avec!». Ce qui était plutôt vrai en termes d'argent. Mais ce qui n'empêchait pas non plus ce paysan, comme tant d'autres individus des villages et même des villes de Roumanie, de continuer à travailler à perte pour avoir ses pommes de terre. À l'autre extrême, nous avons pu voir à Voineşti, par exemple, un tracteur tout neuf, dont le propriétaire était très fier, mais qui restait inutilisé et orné de fleurs dans la grange : "l'inventaire supérieur » était réduit au titre de capital symbolique.

D'une manière plus générale, au fameux précepte de Franklin qui dit que «celui qui tue une truie assassine tous ses descendants jusqu'à la millième génération », les paysans vont répondre plutôt comme cette brave femme qui se demandait " pourquoi garder le veau si une vache nous suffit pour les besoins de la famille? ». Dans le même sens, mais dans un autre contexte, on peut citer aussi l'exemple suivant donné par Creed : dans un combinat métallurgique de Bulgarie, on a décidé de passer au travail en brigade et de payer les gens en fonction de leur production collective. 
50 La production a augmenté un petit peu, mais les ouvriers n'étaient pas motivés pour continuer à maximaliser la production comme prévu. Etant donnée la plus grande maitrise qu'ils avaient de leur programme de travail, les membres de la brigade ont coopéré pour finir la même quantité de travail en moins de temps, de manière à avoir plus de temps pour leur travail agricole personnel. Comme me l'expliquait l'un d'entre eux : « Nous (la brigade), on se rassemble et on décide combien on doit gagner par mois pour les pièces qu'on fait, d'habitude c'est environ 300 leva, puis on voit combien de pièces on doit faire pour avoir cet argent et puis on en fait autant. (...) On fait tout comme le gouvernement, avec un plan. On fait un plan et puis on le réalise ! ${ }^{24}$

51 Sauf que la logique de ce plan était opposée à celle du plan de l'État...

52 Ce rapport au travail et à ses bénéfices est « rationnel » ou « irrationnel » selon les critères qu'on adopte. En tout cas, on peut et on doit se poser la question de savoir pourquoi cette quantité de travail et non pas une autre? Et la raison reste incompréhensible, tant qu'on juge le travail seulement comme moyen de production, par rapport exclusif à ses résultats objectifs et objectuels, c'est-à-dire sans prendre en compte le fait qu'il ne suppose pas seulement un "plus d'avoir », mais aussi un "plus d'être ». Or le travail est justement cela : le rapport entre «ce qu'on est » et « ce qu'on a » à travers "ce qu'on fait », ou, pour simplifier, un rapport entre statut et biens. Le niveau désirable du travail est donc déterminé par la double nature de ses bénéfices, les biens qu'on compte en tirer et le statut qu'on veut acquérir. Dans cette perspective, on peut dire que l'ethos propre à la maisnie est défini par la double limitation d'un plafond maximal de la valeur des biens (pas plus de richesse) et d'un plafond minimal de la valeur des personnes (pas moins de statut).

biens limitation supérieure

54 statut limitation inférieure

Autrement dit, les individus qui partagent ce type d'ethos organisent leur travail de manière à avoir ni plus de biens, mais ni moins de statut que la norme coutumière partagée. Ils vont renoncer à leur travail, même si cela peut leur apporter plus, dès que ce plus n'est pas considéré nécessaire et donc motivé, mais ils vont continuer à travailler, même si cela leur apporte moins, tant que ce moins reste nécessaire pour leur valeur personnelle.

Statut et biens vont donc de pair dans et par le travail, qui est leur condition commune de réalisation, se trouvant ainsi embedded dans cette complémentarité de l'être et de l'avoir. Sa raison ne se trouve pas - et ne doit pas être cherchée - seulement du côté des biens qu'il produit, car ces biens ne sont pas indépendants de la valeur des personnes qui les possèdent.

Mais il ne faut pas se méprendre. Le membre de la maisnie (le gospodar) est dans son travail, mais il n'y est pas entièrement et exclusivement. «Être à l'œuvre ", il est aussi " être désoeuvré »: prendre son temps, le " gaspiller ", se vouer à des activités nonproductives est tout aussi important que se mettre au travail. Du riche calendrier des jours fériés aux causettes quotidiennes, le «désoeuvrement» est le complément nécessaire de cet ethos du travail, à tel point que de nombreux jeunes ouvriers ont préféré, du temps du communisme, un travail moins biens payé, mais plus permissif au niveau du programme, à un travail mieux payé, mais trop «strict » ${ }^{25}$. Ce " gaspillage " n'est irrationnel que du point de vue d'une économie qui se donne comme seule raison, la productivité du travail. 
oeut conclure qu'il y a un ethos spécifique de la maisnie, qu'on peut considérer, à la limite, comme «traditionnel ", et qui est partagé par l'ensemble des acteurs de cette dynamique qu'on a essayé d'invoquer. Avec des variations locales et individuelles des valeurs absolues des "plafonds» supérieur et inférieur, le rapport en tant que tel semble faire la règle. Il en résulte une différenciation sociale relativement faible (mais non négligeable) et une relative indifférence par rapport aux exigences d'un développement économique indéfini. De toute évidence, on n'est pas dans un ethos protestant, qui, en prêchant la double maximisation des biens et du statut par un travail indéfini, a inventé l'être à l'oeuvre et le temps compact d'où le désoeuvrement a été banni.

Par ailleurs, on n'est pas non plus dans une pureté absolue de l'ethos. On peut saisir à tout moment des variations et même des glissements vers d'autres types d'ethos. Ainsi, par exemple, un villageois nous déclarait que «je ne veux pas travailler comme un idiot, mais je ne désire pas être un paysan riche (chiabur) non plus ». On peut parler dans ce cas-là d'un ethos de la subsistance: la limitation des richesses y est encore, mais le travail se retrouve dégradé de condition de statut en instrument de subsistance. Instrumentalisé, le travail peut entrer aussi dans un ethos hédoniste : le moins de travail possible pour le plus de bénéfice possible. Il n'empêche que l'ethos de la maisnie, tel qu'on vient de le décrire, garde une spécificité et une efficacité importantes dans la société roumaine actuelle - ce qui ne signifie pas, bien sûr, qu'il serait spécifique d'une manière quelconque à la société roumaine.

\section{La maisnie diffuse et sa raison sociale}

60 L'obsession des maisons avec beaucoup de chambres (bien plus que nécessaire et qu'on n'utilise jamais), des maisons imposantes (case de fală) qu'on a déjà évoquée à propos de Pucheni, m'a toujours fasciné. On les retrouve partout dans le pays, à tel point qu'on peut parler d'un phénomène social (même s'il n'est pas, bien sûr, général).

La tendance existait déjà du temps du communisme, mais les restrictions idéologiques de «l'égalité sociale » étaient telles qu'elles la gardaient sous un contrôle strict: un habitant de Pucheni, par exemple, qui a voulu introduire un ascenseur dans sa maison à deux étages, a fini en prison. Par ailleurs, « la maison de l'au-delà entraînait des dons funéraires - faits souvent du temps de la vie du futur bénéficiaire - toujours aussi extravagants par rapport aux ressources ${ }^{26}$ et aussi «irrationnels», mais moins « visibles »- et donc contrôlables - ici-bas.

Après la chute du communisme, la visibilité sociale n'est pas seulement permise, mais elle semble emportée par une volonté réparatoire frénétique. Pour l'expliquer et pour comprendre la «raison » de ces constructions «irrationnelles", on peut parler, bien sûr, de "valeur symbolique " et de "visualisation du statut", mais ce serait, me semble-t-il, aller un peu vite. De toute évidence, ce comportement constructif n'est pas isolé, mais fait organiquement partie de l'ensemble des stratégies qu'on a essayé de présenter. Alors, pourquoi tout cela, quelle est la « raison sociale » de cet ensemble de stratégies qu'on vient d'appeler maisnie diffuse? Quelle est la « raison » qui fait que ces choix stratégiques sont « rationnels » et non pas « irrationnels », comme ils en donnent quelquefois l'impression? Ce n'est pas, en tout cas, en faisant appel à tout ce cortège de non-concepts que suppose l'image évolutionniste de la "transition » (une économie qui n'est pas encore de marché, un système politique qui n'est pas encore tout à fait 
démocratique, une société «balkanique » qui n'est pas à proprement parler civilisée, etc.) qu'on va réussir à trouver une réponse !

Les sciences sociales utilisant un modèle fins-moyens requièrent pour passer des prémisses de leurs modèles à des conséquences testables, que les hommes visent ou maximisent quelque chose, peu importe quoi. L'ennui est qu'elles sont prises en fourchette: si elles choisissent des buts correspondant à quelque réalité observable, ceux-ci sont si complexes et diffus qu'ils défient tout calcul ou toute efficacité instrumentale; si elles choisissent des buts qui permettent de tels calculs, ils n'évoquent que de très loin des buts réels ${ }^{27}$.

Le problème est que, "dans la plus grande partie de leur vie, les hommes ne maximisent rien du tout, ni ne cherchent à atteindre un but concrètement identifiable, mais tiennent tout simplement à être intégrés, ou demeurer dans une pièce qui se déroule $\aleph^{28}$. (Quand il se pose la question de savoir pourquoi les gens ont intérêt à faire ce qu'ils font, Bourdieu commence lui aussi par définir l'intérêt par le fait d'être dans le jeu, être investi dans le jeu ${ }^{29}$ ). Pour nous convaincre, Gellner va plus loin et nous propose le test mental de G.E. Moore : imaginez, dit-il, la poursuite d'un but quelconque dans la situation d'un isolement total. Avoir de l'argent, des relations, du prestige, faire des excursions ou collectionner des timbres deviennent dans ces circonstances autant de buts ridicules en tant que « buts en soi de la vie»!

Revenant aux choix stratégiques qui nous occupent, il semble encore moins que leur finalité puisse être seulement le bien-être, mais plutôt quelque chose du genre biens + être, pour ainsi dire. Mais introduire l'être dans les équations économiques risque de faire dérailler toute la machine mathématique. Être, qu'est-ce que cela veut dire?

Les hommes "tiennent tout simplement à être intégrés ", nous dit Gellner. Peut-être doit-on revenir alors à Aristote et à son idée de zoon politikon. De ce point de vue, l'élément « premier » n'est ni l'individu, ni la collectivité, mais la nature collective de l'individu. Gusti disait, dans ce sens, que «l'homme n'est pas social parce qu'il vit en société, mais parce que la société vit en lui ${ }^{30}$. Dans une toute autre perspective, Piaget s'est efforcé dans l'ensemble de son oeuvre de combattre l'adultocentrisme qui voit dans l'enfant un homunculus, un individu en miniature, à l'image des adultes, qui va passer son temps à grandir. Pour la psychologie génétique (et pas seulement), le développement psychique se fait à partir d'un adualisme initial, une non-différenciation entre moi et non-moi et consiste en une individualisation progressive par assimilations et accommodations successives. En reprenant à notre compte ces idées et ajoutant celles des théories de l'identification ${ }^{31}$, on arrive ainsi à définir l'identité individuelle comme synthèse conflictuelle entre identifications et différenciations réalisées dans des contextes sociaux spécifiques et changeants. De ce point de vue, l'individu n'est pas celui qui s'intègre d'une manière progressive et variée dans le monde, mais celui qui se différencie d'une manière progressive et variée par rapport au monde, sans jamais y aboutir entièrement. L'unité d'analyse ne doit plus être alors cette entité première qu'on appelle l'individu, mais l'antagonisme dynamique $e^{32}$, individuel et de groupe, entre identifications et différenciations. C'est ce qu'on a choisi d'appeler la constante relationnelle $(\mathrm{k})$ :

$\mathbf{R}(\mathrm{I})+\mathbf{R}(\mathrm{D})=\mathbf{k}$

où $\mathrm{R}(\mathrm{I})=$ relations d'identification et $\mathrm{R}(\mathrm{D})=$ relations de différenciation. $\mathrm{D}$ 'un point de vue plutôt fonctionnel, les premières sont des relations de tous, les autre étant des relations de chacun. Dans le premier cas, on ira vers une homogénéisation de 
l'ensemble relationnel, une unité croissante et «holiste» de ses membres, embedded ainsi dans leurs relations qui les réunissent tous; dans le deuxième cas, la tendance est vers l'hétérogénéisation, la différenciation des relations de chacun. Dans les termes de cette alternative, on peut considérer le don comme étant l'expression des règles relationnelles de tous (le don concerne les groupes, et non pas les individus, précisait Mauss), tandis que le contrat concerne les règles relationnelles de chacun.

De cette nature « collective » ou " politique » de l'individu découle aussi la nécessité de la politique, car cette nature collective de l'individu ne peut être accomplie que d'une manière collective, dans et par la collectivité. On peut (et on doit, à notre avis) considérer alors la politique comme compétition d'une collectivité pour établir la meilleure manière de vivre ensemble. Autrement dit, l'ordre politique concerne les pratiques et les représentations attachées, qui président les moyens et le sens de la vie en commun, le problème politique étant alors celui de la gestion des relations humaines de manière à donner un sens acceptable à la vie en commun des gens participant à ces relations. En fin de compte, il s'agit de la gestion «sensée » de la constante relationnelle.

Cela veut dire tout simplement que l'homme est un être "politique", obligé par sa «nature » même d'exister en relation et donc de sauvegarder, d'une manière ou d'une autre, une " constante relationnelle » définitoire. Par sa nature politique, l'homme ne peut donc être l'individu de l'économie (néo)classique que d'une manière hypothétique et réductrice. «Refusant de reconnaître l'inscription dans le moi d'une part d'humanité commune, le couple constitué par l'individualisme et la théorie néoclassique essaie de fonder l'éthique du comportement de l'homme n'ayant aucune dette envers quiconque. Ce qui fonde la revendication de cette théorie à être reconnue comme le discours de liberté $»^{33}$. Or la " constante relationnelle » suggère justement qu'il reste toujours une part de dette dans toute relation sociale, sans que l'individu soit entièrement endetté dans n'importe quelle relation sociale.

71 Revenant à notre analyse, nous pensons être en mesure de dire que le principal risque social à éviter n'est pas la pauvreté (avoir faim ou froid n'est pas, en soi, un problème social, mais biologique : dans l'isolement, on n'est pas pauvre, on a faim, comme tout animal; la pauvreté devient sociale quand elle est vécue dans la société), mais l'exclusion. Ce qu'on cherche dans la société n'est pas de maximiser quoi que ce soit, mais la garantie minimale de la vie humaine même: celle d'être-ensemble. La raison sociale de nos actes n'est, en fin de compte, que d'éviter la marginalisation ou, en termes positifs, de "rester dans le jeu ", d'avoir une position dans l'ordre social. Le reste est histoire...

72 Par rapport à cette raison sociale, le système de production et celui des relations sont les moyens de se "positionner ", des moyens d'avoir une position (ou économiques) et des moyens d'être en position (ou politiques), les deux étant en stricte interdépendance $\mathrm{du}$ fait de leur but (ou, plutôt, de leur sens) commun. Ensemble, ils composent l'ordre social, contrôlé et légitimé par un Pouvoir (coercitif ou non) et définissant les coordonnées des positions sociales possibles ou le "pays légal ». C'est dès ce moment que peut intervenir une certaine "maximisation" comme aspiration à une position saturée, qui s'identifie à l'idéal légitimé par un certain ordre social et qui peut expliquer alors, au-delà de tout économisme, tant de choix «fondamentalistes » ou autrement dit « irrationnels ». 
73 Mais, comme on le sait, chaque société, dominée par un ordre social (qui, lui aussi, n'est pas réductible à un seul Jeu, mais suppose des jeux différents) porte en soi, d'une manière ou d'une autre, le désordre social ou, au moins, ses prémisses - c'est-à-dire un choix réactif ou alternatif de moyens de se positionner. C'est le cas de la maisnie diffuse qui, de ce point de vue, est un ensemble de stratégies du désordre social par rapport à l'ordre dominant du communisme, voire à celui «transitionnel» du capitalisme. Sa raison sociale reste celle d'éviter à ses membres (en groupe et non pas individuellement) la marginalisation dictée par un système social trop restrictif (le communisme) ou bien par un autre, encore mal en place, n'offrant pas encore des moyens accessibles de positionnement (le capitalisme). Dans les deux cas, l'ensemble des stratégies regroupées sous le terme de maisnie diffuse n'est pas une simple "survivance ", mais le produit des systèmes qui l'ont vue apparaitre. Et la convergence de ces stratégies dans les deux cas suggère la similitude de problèmes sociaux que deux systèmes aussi différents peuvent produire au-delà de leur opposition.

Toute une économie politique de la maisnie diffuse est encore à faire. Mais pour y parvenir, pour donner raison à ces stratégies capables de métaboliser, d'une certaine manière et à une certaine échelle, communisme et capitalisme, d'en être leur complément de désordre et complétant ainsi l'image du «pays réel », toute l'économie politique est, peut-être, à refaire...

\section{NOTES}

1. Garoflid (Constantin), « Regimul agrar în România », in Enciclopedia României, vol. I, 1938.

2. Stahl (Henri), « Organizarea socială a Tărănimii », in Enciclopedia României, vol. I, 1938.

3. Stahl (Paul), «L'organisation magique du territoire villageois roumain », L'Homme, XII (3), 1973.

4. Stahl (Henri), ContribuŢii la studiul satelor devălmaşe româneşti, vol.III, Bucureşti: Ed. Academiei R.P.R., 1965.

5. Ibid.

6. Mihăilescu (Vintilă), Nicolau (Viorica), « Du village à la ville et retour. La maisnie mixte diffuse en Roumanie », Bulletin of the Ethnographical Institute (Beograd), XLIV, 1995.

7. Voir Mihăilescu (Vintilă), Nicolau (Viorica), Gheorghiu (Mircea ), Olaru (Costel), « Blocul între loc şi locuire. Teme şi probleme de etnologie urbană ", Revista de Cercetări Sociale, 1, 1994.

8. Kideckel (David), The Solitude of the Collectivism : Romanian Villagers to the Revolution and Beyond, Ithaca : Cornell University Press, 1993.

9. Hahn (Chris), "Land Tenure and Citizenship in Tazlar», in Abrahams (Ray), ed., After Socialism. Land Reform and Social Change in Eastern Europe, Providence / Oxford: Berghahn Books, 1996.

10. Voir Mesnil (Marianne), Mihăilescu (Vintilă), « La table sans dessus dessous. De l'hospitalité agonistique », La Revue du MAUSS, (12), 1998.

11. Kideckel (David), op. cit.

12. Ibid. 
13. Cf. Mihăilescu (Vintilă), « Două sate în tranziŢie. Tipuri strategice dominante în mediul rural românesc », Revista de Cercetări Sociale, (3), 1996.

14. Voir Mihăilescu (Vintilă), Nicolau (Viorica), art. cit.

15. Stahl (Henri), op. cit.

16. Cf. Mihăilescu (Vintilă), art. cit.

17. Voir à ce sujet Oprescu (Adriana), Les enjeux politiques de la gestion du temps dans la Roumanie des années 80, Mémoire de DEA, Université Libre de Bruxelles, 1998.

18. Creed (Gerald), Agriculture and the Domestication of Industry in Rural Bulgaria, in press.

19. Kideckel (David), op. cit.

20. Creed (Gerald), op. cit.

21. Vulcănescu (Mircea), Prolegomene sociologice la satul românesc, București : Editura Eminescu, 1997.

22. Cf. ibid.

23. Ibid.

24. Creed (Gerald), op. cit.

25. Cf. Kideckel (David), op. cit.

26. Andreesco (Ioana), Bacou (Mihaela), Mourir à l'ombre des Carpathes, Paris : Payot, 1986.

27. Gellner (Ernest), «L'animal qui évite les gaffes, ou un faisceau d'hypothèses », in Birnbaum (Pierre), Leca (Jean), éds., Sur l'individualisme. Théories et méthodes, 1986.

28. Ibid.

29. Bourdieu (Pierre), Raisons pratiques. Sur la théorie de l'action, Paris : Editions du Seuil, 1994.

30. Gusti (Dimitrie), La science de la réalité sociale. Introduction à un système de sociologie, d'éthique et de politique, Paris : Alcan PUF, 1941.

31. Cf. par exemple Bloom (William), Personal Identity, National Identity and International Relations, Cambridge : Cambridge University Press, 1990.

32. Lupasco (Stéphane), Psychisme et sociologie, Paris : Casterman, 1978.

33. Insel (Ahmet), «Une rigueur pour la forme. Pourquoi la théorie néoclassique fascine-t-elle tant les économistes, et comment s'en déprendre ? » , La Revue du MAUSS, (3), 1994.

\section{INDEX}

Index géographique : Roumanie

Mots-clés : Propriété, Système de relations

\section{AUTEUR}

\section{VINTILĂ MIHĂILESCU}

Université de Bucarest 